OPEN ACCESS

Edited by:

Helene Dutartre

UMR 5308 Centre International de Recherche en Infectiologie (CIRI),

France

Reviewed by:

Matteo Biolatti,

University of Turin, Italy

Alina Deshpande,

Los Alamos National Laboratory (DOE), United States

*Correspondence:

Anna Rosa Garbuglia

argarbuglia@iol.it

†These authors have contributed equally to this work

Specialty section: This article was submitted to Virology,

a section of the journa

Frontiers in Microbiology

Received: 07 September 2020 Accepted: 03 November 2020 Published: 25 November 2020

Citation:

Sias C, Guarrasi V, Minosse C Lapa D, Nonno FD, Capobianchi MR Garbuglia AR, Del Porto $P$ and Paci $P$ (2020) Human Papillomavirus

Infections in Cervical Samples From HIV-Positive Women: Evaluation of the Presence of the Nonavalent HPV

Genotypes and Genetic Diversity.

Front. Microbiol. 11:603657.

doi: 10.3389/fmicb.2020.603657

\section{Human Papillomavirus Infections in Cervical Samples From HIV-Positive Women: Evaluation of the Presence of the Nonavalent HPV Genotypes and Genetic Diversity}

\author{
Catia Sias ${ }^{1+}$, Valerio Guarrasi2t, Claudia Minosse', Daniele Lapa', Franca Del Nonno ${ }^{3}$, \\ Maria Rosaria Capobianchi' ${ }^{1}$, Anna Rosa Garbuglia ${ }^{1 *}$, Paola Del Porto ${ }^{4}$ and Paola Paci ${ }^{2}$ \\ ${ }^{1}$ Laboratory of Virology, Lazzaro Spallanzani National Institute for Infectious Diseases, IRCCS, Rome, Italy, ${ }^{2}$ Dipartimento di \\ Ingegneria Informatica, Automatica e Gestionale "A. Ruberti", Sapienza Università di Roma, Rome, Italy, ${ }^{3}$ Laboratory \\ of Pathology, Lazzaro Spallanzani National Institute for Infectious Diseases, IRCCS, Rome, Italy, ${ }^{4}$ Department of Biology \\ and Biotechnology "C. Darwin”, Sapienza University, Rome, Italy
}

Non-nonavalent vaccine (9v) Human papillomavirus (HPV) types have been shown to have high prevalence among HIV-positive women. Here, 1444 cervical samples were tested for HPV DNA positivity. Co-infections of the 9v HPV types with other HPV types were evaluated. The HPV81 $L 1$ and $L 2$ genes were used to investigate the genetic variability of antigenic epitopes. HPV-positive samples were genotyped using the HPVCLART2 assay. The $L 1$ and $L 2$ protein sequences were analyzed using a self-optimized prediction method to predict their secondary structure. Co-occurrence probabilities of the 9v HPV types were calculated. Non9v types represented $49 \%$ of the HPV infections; $31.2 \%$ of the non9v HPV types were among the low-grade squamous intraepithelial lesion samples, and $27.3 \%$ among the high-grade squamous intraepithelial lesion samples, and several genotypes were low risk. The co-occurrence of $9 \mathrm{v}$ HPV types with the other genotypes was not correlated with the filogenetic distance. HPV81 showed an amino-acid substitution within the BC loop (N75Q) and the FGb loop (T315N). In the L2 protein, all of the mutations were located outside antigenic sites. The weak cross-protection of the $9 \mathrm{v}$ types suggests the relevance of a sustainable and effective screening program, which should be implemented by HPV DNA testing that does not include only high-risk types.

Keywords: Human papillomavirus, HPV mixed infection, genetic variability, intraepithelial lesions, HPV vaccine

\section{INTRODUCTION}

Cervical cancer is the fourth most frequent cancer in women (Ferlay et al., 2019). Statistical data suggest that almost $99 \%$ of cervical cancers are related to Human papillomavirus (HPV) infections (Walboomers et al., 1999; De Martel et al., 2012). For 70\% of the women who become infected during the first half of their lives, only $10 \%$ of these infections become persistent, and a minority of these progresses to cervical cancer (Woodman et al., 2007). The evolution toward 
intraepithelial lesions is related to HPV genotypes. The International Agency for Research on Cancer has indicated the $15 \alpha$-types as high-risk (HR) genotypes: HPV16, 18, 31, 33, 35, 39, 45, 51, 56, 58, 59, 66, 68, 73, and 82 (Muñoz et al., 2003). These genotypes are responsible of more than $90 \%$ of all cervical cancers (Luostarinen et al., 2018), and they are also involved in anal and oropharynx cancers (Garbuglia et al., 2020). While the lowrisk (LR) types are predominantly involved in the development of genital warts, only HPV6 and HPV11 are responsible for recurrent respiratory papillomatosis. This disease is very difficult to treat, and it often has fatal outcomes (Garbuglia et al., 2020).

In developed countries, the cervical cancer screening program started 40 years ago, and it has contributed to reduced incidence of and mortality from cervical cancers (Peirson et al., 2013). However, the prevalence of invasive cervical cancer (ICC) remains high in Africa, southeast Asia, and eastern Europe, where preventive medicine is not practiced or is ineffective. Indeed, in these geographical areas, the population screening program reaches between $6 \%$ and $8 \%$ of the female population (Sudenga et al., 2013). Therefore, a prophylactic vaccine represents a powerful tool for eradication of cervical cancer.

In 2006, two L1 virus-like particle (VLP) vaccines were licensed: Cervarix, a bivalent vaccine that contains VLPs of the HPV16 and HPV18 HR types, and Gardasil, a quadrivalent vaccine that includes VLPs of the HPV16 and HPV18 HR types, plus the two: HPV6 and HPV11 LR types. These vaccines prevent $70 \%$ of cervical cancers, and the tetravalent vaccine $(4 \mathrm{v})$ can also prevent epidermodysplasia associated to HPV6 and HPV11 infections (Garland et al., 2007). In December 2014, a non-avalent vaccine (9v) was licensed by the United States Food and Drug Administration, and in June 2015, this was also approved by the European Commission (Lopalco, 2016). VLPs of five additional HR types, HPV31, 33, 45, 52, and 58, were included in the 9v. Theoretically, the $9 \mathrm{v}$ can prevent up to $93 \%$ of cervical cancers (Brotherton et al., 2017), with 100\% seroconversion documented, for antibody titers that remain stable over decades (Nygård et al., 2015). Countries that have included high vaccination coverage have obtained $73 \%$ to $85 \%$ reductions in infections by the $9 \mathrm{v}$ HPV types, and $41 \%$ to $75 \%$ decreases in high-grade lesions [cervical intraepithelial neoplasia (CIN) grade 2 or worse] for up to 10 years after vaccination (Drolet et al., 2019). In addition, clinical trials carried out after the vaccine licensing have demonstrated an efficacy of up to $100 \%$ against vulvar and vaginal intraepithelial neoplasia for the bivalent and quadrivalent vaccines administrated to virus-naïve women. Despite these encouraging results, data have appeared in the literature that describe high-grade squamous intraepithelial lesions (HSIL) or cervical tumors linked to HPV types that are not included in the current vaccines. Amaro-Filho et al. (2020) identified HPV73 in cervical cancer, which was previously considered a LR type. HPV73 and HPV56 have also been frequently associated with vaginal intraepithelial neoplasia 2/3, and HPV51 and HPV59 with vulvar intraepithelial neoplasia 2/3, in Japan (Sugase and Matsukura, 1997). In a study carried out among women with HSIL or ICC, $4.5 \%$ of the patients with CIN 2/3, and $3.9 \%$ of those with cervical cancer were positive to one or multiple LR HPV types. HPV54 and HPV73 have been observed in cervical cancers, with HPV42, 54, 62, 67, 70, and 82 reported for CIN2 samples (Siegler et al., 2019). In Korea, HPV56, 68, and 73 were detected in adenocarcinoma, and HPV59 in squamous cell carcinoma (Park et al., 2019), while HPV82 was reported as the most frequently observed HPV type in HSIL in Oman (AlLawati et al., 2020). Although the 9v has shown broad efficacy against the five additional HR types (Toft et al., 2014), it is not known whether it can cross-protect against all non-vaccine HPV types. The lifespan of the cross-neutralizing antibodies following HPV immunization is also not known (Toh et al., 2019). In particular, among HIV positives, the cross-neutralizing antibody titers were lower than those of HIV-negative people (Weinberg et al., 2012).

These aspects are particularly relevant among HIV-infected women, who have a high rate of HPV infections and harbor multiple HPV infections more persistently, in comparison to HIV-negative women (Palefsky et al., 1999; Garbuglia et al., 2012). In a 4-year follow-up study on HIV-positive women who were vaccinated with the $4 \mathrm{v}$, incidence and persistence of HPV51 and HPV39 types were observed. This suggests that previous data on the $4 \mathrm{v}$ cross-neutralization activities described in HIV-negative women did not extend to HIV-positive women (Toh et al., 2019).

The aim of the present study was to determine the prevalence of HPV types included and not included in $9 \mathrm{v}$ in low-grade squamous intraepithelial lesions (LSIL) and HSIL among HIVpositive women. Based on these observations, a further aim was to determine the probability that the $9 \mathrm{v}$ genotypes co-exist with the other HPV types that are circulating among HIVpositive women in Italy. Furthermore, the L1 and L2 genes were analyzed for single infections (SIs) and multiple infections (MIs), to investigate the genetic variability of the antigenic epitopes in these two groups (i.e., SIs, MIs).

\section{MATERIALS AND METHODS}

\section{Study Population}

This was a cross-sectional study that included HIV-positive women who had been screened for HPV-related diseases at the gynecology Outpatient Service of the "L. Spallanzani" National Institute for Infectious Diseases (IRCCS, Rome). The Institutional Ethical Committee approved the study protocol (n. 42/13). All of the methods were carried out according to the ethical standards of the Institutional and National Research Committee and the Declaration of Helsinki.

Cervical cell samples were obtained using a cytobrush, and were preserved in $2 \mathrm{~mL}$ phosphate-buffered saline [24], and stored in ice until they were delivered to the Laboratory of Virology, where they were processed. Cervical smears were prepared for cytological examination (Pap-tests) concomitant with sampling for HPV testing. Cytological diagnosis was formulated according to the 2001 Bethesda system (Solomon et al., 2002), and classified as normal or abnormal (diskaryosis) findings. This latter group included atypical squamous cells of undetermined significance, LSIL, or HSIL. All SIL cases were confirmed by colposcopies and biopsies. 


\section{HPV-DNA Analysis}

Approximately $1.8 \mathrm{~mL}$ cervical swabs samples were centrifuged at $1,500 \times g$ for $5 \mathrm{~min}$. The pellet was resuspended in $1.5 \mathrm{~mL}$ phosphate-buffered saline, and $20 \mu \mathrm{L}$ proteinase $\mathrm{K}$ (Qiagen, Hilden, Germany) was added, with the samples then incubated at $56^{\circ} \mathrm{C}$ for $10 \mathrm{~min}$. An aliquot of $800 \mu \mathrm{L}$ was used to extract the nucleic acids (Qiasymphony automatic instrument; Qiagen, Hilden, Germany), according to the manufacturer instructions. The residual samples were immediately frozen and stored at $-80^{\circ} \mathrm{C}$ until used for further investigations.

All of the samples were tested for amplifiable DNA using $\beta$ globin primers (Saiki et al., 1985; Sias et al., 2019), and HPV was detected using the MY09/11 primers, as previously described (Garbuglia et al., 2012). The PCR products were visualized by $1.8 \%$ agarose gel electrophoresis with ethidium bromide staining. HPV-positive samples were genotyped (HPVCLART2 assay; Genomica, Madrid, Spain; Garbuglia et al., 2012). This assay is based on nucleic acid amplification technology followed by visualization in low-density microarrays of the HPV PCR product. This system detected $35 \mathrm{HPV}$ types: $15 \mathrm{HR}$ types (HPV16, 18, 31, 33, 35, 39, 45, 52, 56, 58, 59, 66, 68a and 68b, 73, and 82), 15 LR types (HPV6, 11, 41, 42, 43, 44, 54, 62, 71, 72, 81, $83,84,85$, and 89), and four potential carcinogenic types (HPV26, 51, 53, and 70). Samples, that could not be typed by Genomica were analyzed by Sanger sequencing, as described in Sias et al. (2013). Briefly, the MY09/11 PCR products were purified with Qiaquick for PCR products (Qiagen, Hilden, Germany) and directly sequenced using a genetic analyzer (AB3130; Applied Biosystems, Life Technology, Forster, CA, United States). The HPV types were identified and quantified by comparison of the DNA sequences with those available in GenBank, through the BLAST server (BLAST, 2020), and they were classified according to De Villiers (De Villiers, 2013).

\section{L1 and L2 Sequences}

To study the L1 genetic variability of HPV81, we selected a subset of HPV81 SIs $(n=6)$ and a subset of HPV81 MIs $(n=4)$. HPV81 was chosen because it has been frequently observed in association with LSIL and HSIL grade lesions (Minosse et al., 2010; Annunziata et al., 2018; Ge et al., 2019), though it is considered a LR type, and little information is available on its genetic variability. The PCR to amplify the $L 1$ region was based on two PCRs that were combined to obtain the full length of the $L 1$ sequences. All of the primer mixes were directed against highly conserved motifs of the HPV81 genome. L1 region mix A generated fragments of approximately $850 \mathrm{bp}$ in length, while the mix B gave a PCR product of 950 bp (Table 1). Each mix consisted of $50 \mu \mathrm{L} \mathrm{1x}$ TaqGold buffer (TermoFisher Scientific, United States), $1.5 \mathrm{mM} \mathrm{MgCl} 2,0.2 \mathrm{mM}$ each dNTP, $0.5 \mu \mathrm{M}$ each primer, $1.5 \mathrm{U}$ TaqGold polymerase (TermoFisher Scientific, United States), and $10 \mu \mathrm{L}$ DNA extracted from cervical samples. The assay conditions were: $15 \mathrm{~min}$ at $94^{\circ} \mathrm{C}$, and 35 cycles: $94^{\circ} \mathrm{C}$ $40 \mathrm{~s}, 58^{\circ} \mathrm{C}$ for $45 \mathrm{~s}$, and $72^{\circ} \mathrm{C}$ for $115 \mathrm{~s}$, followed by a final extension at $72^{\circ} \mathrm{C}$ for $10 \mathrm{~min}$.

The L2 gene was amplified by nested PCR amplification using the L2 outer sense and antisense primers (round I), and the
L2 inner sense and antisense primers (round II; Table 1). The composition of the reaction mixture was as used for the L1 gene. After a denaturation step at $94^{\circ} \mathrm{C}$ for $15 \mathrm{~min}$, amplification reactions were for 35 cycles for each round, and consisted of $94^{\circ} \mathrm{C}$ for $45 \mathrm{~s}, 56^{\circ} \mathrm{C}$ for $45 \mathrm{~s}$, and $72^{\circ} \mathrm{C}$ for $90 \mathrm{~s}$. Amplification products were purified (QIAquick PCR purification spin kits; Qiagen $\mathrm{GmbH}$, Hilden, Germany) and sequenced directly using Big Dye Terminator Cycle Sequencing Ready Reaction kits, and analyzed using a sequencing system (AB3130; Applied Biosystems, Forster, CA, United States). The PCR and sequencing were performed in duplicate.

All of the sequences were aligned with an HPV81 prototype sequence (GenBank accession number, AJ620209). The sequences of the L1 and L2 genes were submitted to the NCBI GenBank database, and were assigned accession numbers (MT547553 - MT547561).

Amino-acid sequences were aligned using CLUSTALW (Thompson et al., 1997). We used a self-optimized prediction method (NPSA, Combet et al., 2000; Prabi, 2020) to estimate the $\beta$-turn structure. $\beta$-Turns are very frequently the sites of interactions in proteins and peptides, both because they are topologically biased to occur on the surfaces of proteins, and because their structures present the side chains of corner residues, which are optimal for molecular recognition. The Hamming distance (Hamming, 1986; Farci et al., 2000) was calculated using online software (Hacksparrow, 2019). The Pearson distance and phylogenetic tree were calculated using the MEGA10 software (Kumar et al., 2018). The phylogenetic tree of the L1 protein was calculated according to Anisimova (Anisimova and Gascuel, 2006), using online software (Phylogeny, 2020).

\section{Co-occurrence Probability Determination}

To compute the co-occurrence probability between $9 \mathrm{v}$ types and the other HPV types detected by Genomica, we evaluated the empirical (or experimental) probability of an event that is an "estimate" that an event will occur based upon how often the event occurred after collecting data from an experiment in a large number of trials (Bickel and Doksum, 1977; Durrett, 1996). This type of probability is based upon direct observations. Each observation in an experiment is called a trial. The empirical probability is given by Eq. (1; Bickel and Doksum, 1977; Durrett, 1996):

$$
P(E)=\frac{\text { number of times the event occurs }}{\text { total number of trials }} .
$$

In our analysis, the event $E$ is the co-occurrence between the reference $9 \mathrm{v}$ non-avalent genotypes (called genotype $A$ ) and one of the others (called genotype $B$ ), and the total number of trials is the number of times the reference genotype occurs in the given dataset (Bickel and Doksum, 1977; Durrett, 1996). Thus, we called this in our analysis $P(E)$, as the co-occurrence probability of the event $E$. Finally, we defined the complementary probability $C(E)$ of event $E$ as the probability of not observing $A$ given $\mathrm{B}$, as in Eq. (2):

$$
C(E)=1-P(E)
$$


TABLE 1 | Primers used for the amplification of $L 1$ and $L 2$ of HPV81.

\begin{tabular}{|c|c|c|c|c|c|}
\hline Gene & Mix & HPV81-L1/L2 primer & Location (nt)* & Sequence $\left(5^{\prime} \rightarrow 3^{\prime}\right)$ & Amplicon length (bp) \\
\hline \multirow[t]{8}{*}{ L1 } & 1 round $A$ & OuterSense1 & $5647-5663$ & ACCACCGTTCCTITGTC & 930 \\
\hline & & Antisense1 & $6577-6550$ & CTCAGCAGCCATITGTAAATAATCTGGA & \\
\hline & 2 round $A$ & InnerSense1 & $5724-5745$ & AGCCCCTTCTATAGTCCCTTCG & 853 \\
\hline & & Antisense1 & $6577-6550$ & CTCAGCAGCCATITGTAAATAATCTGGA & \\
\hline & 1 round $B$ & OuterSense2 & $6525-6544$ & ATATITGCAATACCACCTGT & 954 \\
\hline & & Antisense2 & $7478-7458$ & CAATACACATATAAATACAAC & \\
\hline & 2 round $B$ & InnerSense2 & $6550-6577$ & TCCAGATTATITACAAATGGCTGCTGAG & 928 \\
\hline & & Antisense2 & $7478-7458$ & CAATACACATATAAATACAAC & \\
\hline \multirow[t]{4}{*}{ L2 } & 1 round & OuterSense & $4390-4410$ & CTGCACATTGTITGTACTAT & 1524 \\
\hline & & Antisense & $5914-5895$ & AGACACAGGTGTGGGAGGCA & \\
\hline & 2 round & InnerSense & $4437-4457$ & TACATTGTATACTCTATTGTA & 1477 \\
\hline & & Antisense & $5914-5895$ & AGACACAGGTGTGGGAGGCA & \\
\hline
\end{tabular}

*Nucleotide positions refer to the prototype AJ620209.

Thus, the complementary probability is maximal [i.e., $C(E)=1$ ] when $P=0$, which means that the presence of A rules out the possibility to have $B$.

\section{Least Squares Method}

To determine correlations between the co-occurrence and genotype distance (both Hamming and Pearson genetic distances), we used least squares analysis, which is a standard approach in regression analysis (Feller, 1971; Bickel and Doksum, 1977; Kelley, 1994; Bretscher, 1995; Durrett, 1996; Casella and Berger, 2002; Larson and Farber, 2003; Moore and McCabe, 2003). To assess the strength of the correlations, the $R^{2}$ index was evaluated. $R^{2}$ is a statistical measure that represents the proportion of the variance for a dependent variable that is explained by an independent variable or variables in a regression model. Whereas correlations explain the strength of the relationships between independent and dependent variables, $R^{2}$ explains to what extent the variance of one variable explains the variance of the second variable. So, if $R^{2}$ of a model is 0.50 , then approximately half of the observed variation can be explained by the model inputs. An $R^{2}$ of 1 indicates that the regression predictions perfectly fit the data (Feller, 1971; Kelley, 1994; Bretscher, 1995; Casella and Berger, 2002; Larson and Farber, 2003; Moore and McCabe, 2003). The regression linear model was calculated only on the points where the co-occurrence probability was greater than zero. Finally, we computed the 95\% confidence intervals of the least squares linear regression line. The $95 \%$ confidence interval is the range of values for which you can be $95 \%$ certain contains the true mean of the population. As the sample size increases, the range of the interval values narrows, meaning that you know that mean with much more accuracy compared with a smaller sample (see Supplementary Data 1).

All of the computations were completed using open-source tools, with Python version 3.6 (Python, 2020); in particular, and with the following libraries: pandas (Pandas, 2020), numpy (NumPy, 2020), scipy (Scipy, 2020), matplotlib (matplotlib, 2020), and seaborn (Seaborn, 2020).

\section{RESULTS}

\section{HPV Prevalence and Cytological Findings}

Between 2008 and 2012, 1,444 cervical samples from HIVpositive women were screened for HPV DNA. The median age of the women was 52.63 years (range, 27-89 years), and none of them had been vaccinated. A total of 602 samples were HPV DNA positive (41.7\%) and 21/602 (3.5\%) were $\beta$-globin PCR negative. Also, 336/602 (55.8\%) were HPV SI, and 266 (44.2\%) were HPV MI. For eight samples, the Genomica CLART 2 Array failed to detect any HPV genotypes. These samples were submitted to Sanger sequencing, which allowed the identification of their HPV types: HPV13, HPV55, HPV69, HPV102 $(n=2), \operatorname{HPV} 118$, and HPV120 $(n=2)$. Overall, 9v types (HPV6, 11, 16, 18, 31, 33, 45,52 , and 58) were found in 51.0\% (307/602) of HPV-positive samples: $39.3 \%$ (132/336) were SI and 65.8\% (175/266) were MI. No 9v types represented $49.0 \%$ (295/602) of HPV infections, $69.2 \%(204 / 295)$ of which were SI, and $30.9 \%(91 / 295)$ were MI (Figure 1).

The women were also screened for cervical lesions. Cytological findings were available for 1326 patients. A total of 286 patients $(21.6 \%)$ had cervical dysplasia of any grade (i.e., LSIL or HSIL). No intraepithelial cervical cancer was seen. Here, 97 patients showed abnormalities for atypical squamous cells of undetermined significance or atypical glandular cells of undetermined significance, whereas 911 patients had normal cytological results. LSIL was observed in 231 specimens, HSIL in 55 specimens. Among the LSIL findings, 74 samples (32.2\%) harbored HPV types not included in 9v. Fifteen HSIL were non9v HPV types (27.3\%); nine were SI, and six were MI (Figure 2). Considering SI and intraepithelial lesions, LR HPV42, 54, 72, and 84 were detected only in LSIL, HPV83 only in HSIL, and HPV61, 62, 81 in both (Figure 2A). Five MI, which harbored only LR HPV types, were linked to LSIL (Figure 2B).

\section{HPV Co-occurrence}

Through an analysis of HPV MIs, we investigated the probabilities of co-occurrence of the HPV types included in the 9v (HPV6, 11, 16, 18, 31, 33, 45, 52, and 58) with the HR and LR 


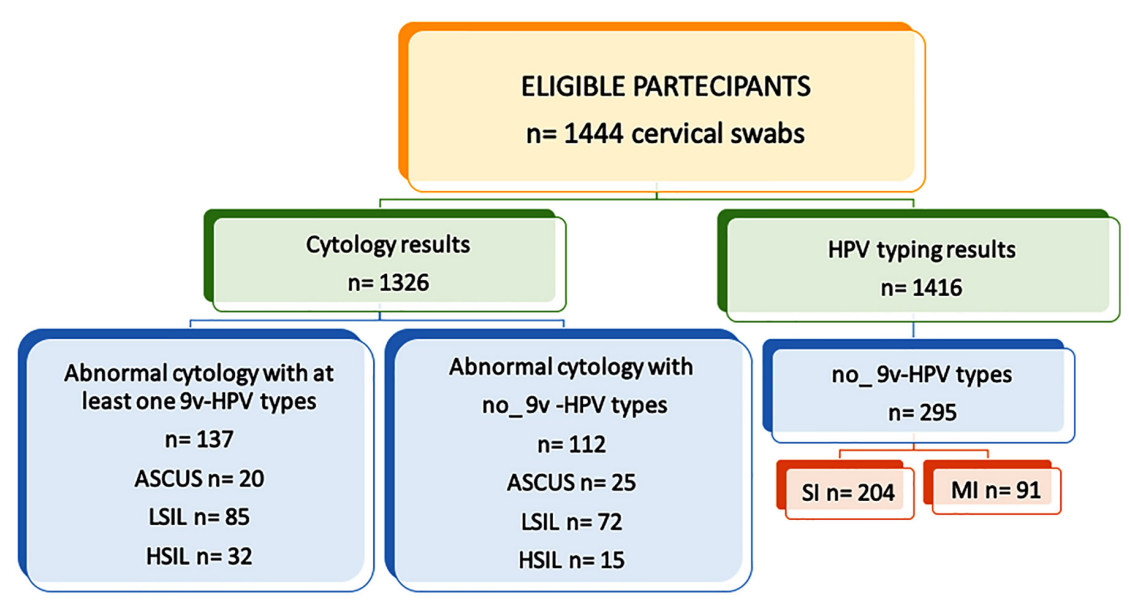

FIGURE 1 | Study design flow chart. Human papillomavirus (HPV) DNA testing results and cytological findings. 9v: nonavalent vaccine; ASCUS, atypical squamous cells of undetermined significance; LSIL, low-grade squamous intraepithelial lesion; and HSIL, high-grade squamous intraepithelial lesion.

HPV types detected by the HPV Genomica CLART2HPV-assay in cervical samples of HIV-positive women. Each of the panels in Figure 3 corresponds to a genotype subtype of the 9v HPV types indicated above the panel (reference genotype) and the further $26 \mathrm{HR}$ and LR HPV types. Each panel depicts the probability $P$ ( $y$-axis) that a specific HR or LR HPV genotype (each column in each panel, $x$-axis) co-exists with the reference genotype. In each panel, the red columns represent HPV genotypes that belong to the same species as the reference genotype, and the blue columns represent HPV genotypes belonging to different classes from the reference genotype. $P$ represents the probability that two different HPV types can occur together. Thus, a null probability between genotype A given $\mathrm{B}$ means that the presence of A rules out the possibility to have B. In this sense, we say that the null probability corresponds to perfect cross-protection, or even that the probability of co-occurrence is the minima.

For instance, the genotype subtype HPV6 does not occur with HPV26, 35, 40, 43, 54, 73, 85, and 89; whereas the $\alpha 9 \mathrm{HR}$ types (HPV16, 31, 33, 52, and 58) show similar probabilities of co-occurrence with the other $\alpha 9 \mathrm{HPV}$ (HPV35), or with other HPV genotypes that belong to different species. For instance, the probability of HPV35 in the presence of HPV16 is 0.077 which is the same as the probability for HPV59. The same observation holds for $\alpha 7$ species (HPV18, 45) that have the same probability of co-occurrence with $\alpha 7$ (HPV59, 68, 70) or with other $\alpha$-species [i.e., C $($ HPV18 $\mid$ HPV59 $)=\mathrm{C}($ HPV18 $\mid$ HPV81 $)=1-0.176=0.8$ ]

The analysis of the results here allows us to conclude that infection by each of the 9v HPV types is associated with the presence of a specific set of HPV genotypes. In particular, these data suggest that genotype HPV6 might protect from HPV26, 35, 40, 43, 54, 73, and 85 infections; HPV 11 from HPV26, 35, 39, 40, 43, 51, 54, 56, 68, 71, 73, 81, 85, and 89; HPV16 from HPV26, 40, 43, 44, 54, and 85; HPV18 from HPV26, 35, 39, 40, 42, 43, 54, 73, 85, and 89; HPV31 from HPV26, 39, 40, 43, 54, 56, 68, 72, 85, and 89; HPV33 from HPV26, 35, 39, 43, 44, 66, 68, 71, 81, 85, and 89; HPV45 from HPV26, 35, 39, 43, 44, 51, 53, 56, 61, 62, 68, 70, 71, 72, 73, 81, 82, 83, 84, 85, and 89; HPV 52 from HPV26, 40, 43, 51,
68, 71, 73, 85, and 8, and HPV58 from HPV26, 40, 43, 71, and 85. The evidence that infection by at least one of the 9v HPV types is never associated with the presence of HPV26, 35, 40, 43, 54, and 73 can lead to speculate that the 9v HPV types might completely protect from co-infections by the six HPV genotypes.

We then evaluated whether the co-occurrence probability correlates with the genotype distance. We used the least squares regression method to quantify this correlation. In Figure 4, each panel corresponds to a single genotype subtype of the $9 \mathrm{v}$. Each panel shows the scatter plot between the co-occurrence probability ( $y$-axis) and the genetic distance ( $x$-axis), where the dots represent the 26 high and low risk HPV genotype subtypes. The co-occurrence probability is the same as for Figure 3, while the genetic distances were calculated using the Poisson method, which includes insertion and deletion. The blue line represents the least squares linear regression line with relative confidence intervals at $95 \%$, the Pearson correlation and the $R^{2}$ represents the linear regression model fitting index used to quantify how well the co-occurrence probability correlates with the genetic distance. In each panel, the fitting model was built considering only the genotypes with a non-null probability (Figure 4, green dots) of simultaneous occurrence with the reference genotype. Red dots correspond to genotypes with null probability of cooccurrence with the reference genotype. Figure 5 shows the same analysis in which the Hamming distances were used to quantify the distances between different genotypes. There were no linear dependencies between co-occurrence and genetic distance (Figures 4-6).

\section{L1 and L2 HPV81 Sequence Analysis}

A total of four HPV81 L1 SI (Pt627, Pt635, Pt807, and Pt822) and two HPV81 MI (Pt798, Pt802) were successfully identified (Figure 7). Five HPV81 L1 sequences were added to the analysis, from previously described MI (Pt211, Pt363, Pt422, Pt574, and Pt644; Minosse et al., 2010). The DNA sequence analysis showed 51 single nucleotide substitutions, of which 35 represented nonsynonymous changes (Figure 7A; Supplementary Figure 1). The 


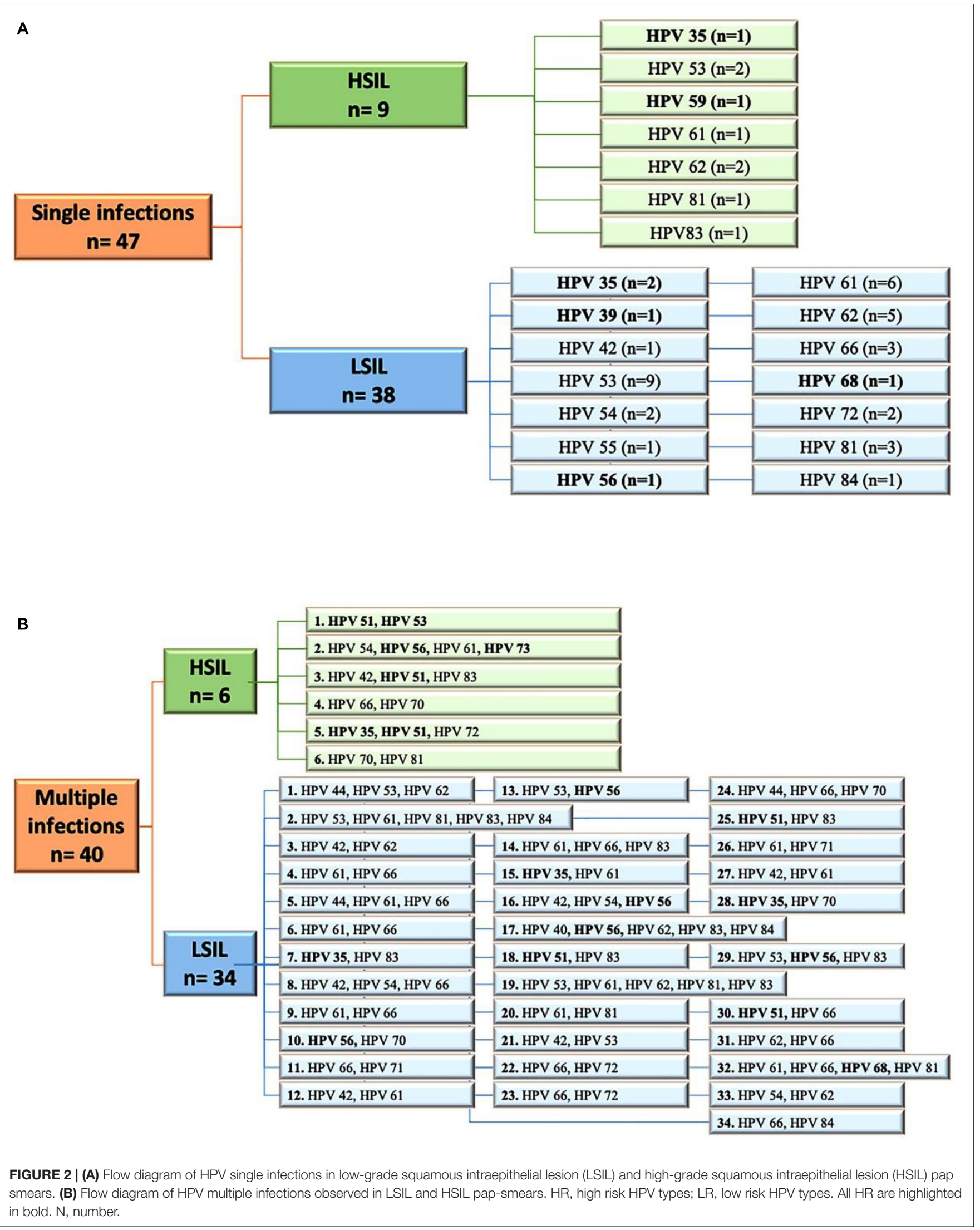



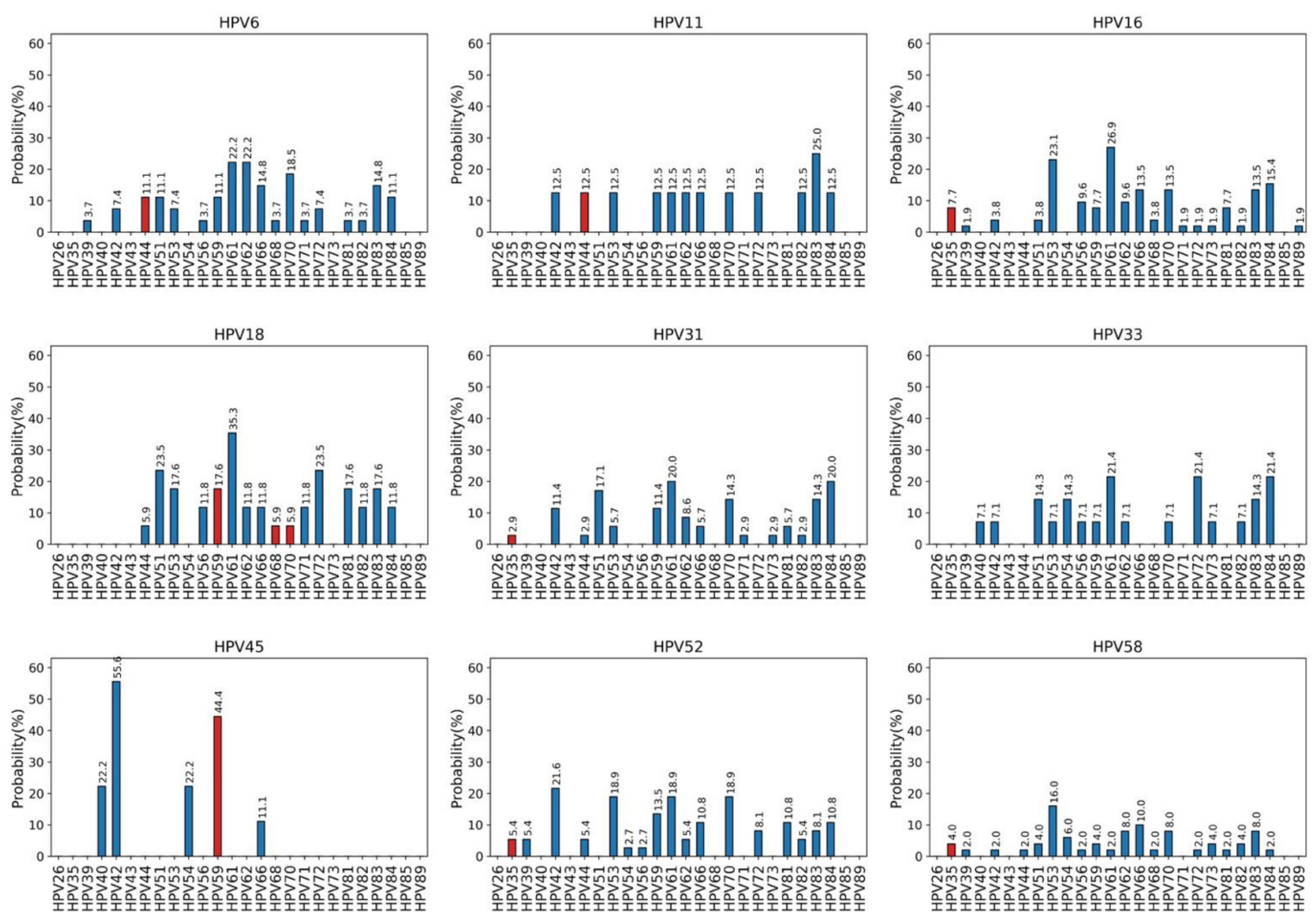

FIGURE 3 | Co-occurrence probabilities. Each panel corresponds to a genotype subtype of the nonavalent vaccine indicated above the panel (reference genotype), and the columns correspond to 26 high-risk and low-risk HPV genotypes detected by the CLART2 array. In each panel, red columns represent HPV genotypes belonging to the same species of the reference genotype, and blue columns represent HPV genotypes belonging to different classes from the reference genotype.

G7332A (R520K) and A7334T (T521S) changes were observed in all of the strains, and thus they can be considered as polymorphisms. Variations of the L1 surface-exposed region, which is considered to be involved in immune recognition, were analyzed (Christensen et al., 2001; Bishop et al., 2007). Among HPV81 MI, one of the seven patients (Pt422) showed an amino-acid substitution within the BC loop (N75Q), while five of the seven patients showed an amino-acid substitution in the FGb loop (T315N); no mutations were observed in HPV81 SI. All of the other amino-acid variations were randomly distributed along the L1 sequence, and here was no evidence of a premature stop codon. The $\beta$-turn prediction showed significant variability compared to the prototype only in MI (Supplementary Table 1). All of the nucleotide changes and amino-acid mutations are described in Supplementary Figure 1. Proline and glycine amino-acid changes, which influence the $\beta$-turn stability (Trevino et al., 2007), were detected only in MI (Figure 7A).

The L2 gene was successfully amplified and sequenced in two SI (Pt807, Pt822) and in five MI (Pt211, Pt574, Pt644, Pt798, and Pt802) samples. Eleven nucleotide changes were non-synonymous mutations (Supplementary Figure 1), and they resulted in nine amino-acid substitutions of the 472-amino-acids-long L2 protein (genetic variability, 1.9\%; Figure 7B). All of the mutations were located outside the antigenic sites. The substitution of A350T was observed only in MI, whereas S346P was found only in two HPV81 strains of SI. For the seven HPV variants we sequenced, we observed no evidence of L2 variants that can escape immune recognition after treatment with $9 \mathrm{v}$ (Roden et al., 2000; Gambhira et al., 2007). The RG-1 epitope, which encompasses residues 15-34 in L2 HPV81, showed a high degree of conservation, and only Pt211 showed the I34V substitution. The furin cleavage sites (amino acids 9-12) and DNA binding motif (amino acids 1-12) were identical to the prototype in all of the analyzed specimens. No $\beta$-turn variability was seen in MI or SI L2 protein (Supplementary Table 1). Only Pt807 with a I425V substitution at the $\mathrm{COOH}$ carboxyterminal had a weak increase in $\beta$-turn coefficient (Supplementary Table 1).

\section{DISCUSSION}

HIV-positive women have a considerable risk of developing ICC, and HSIL is considered an AIDS-defining case (Maiman, 1994). 


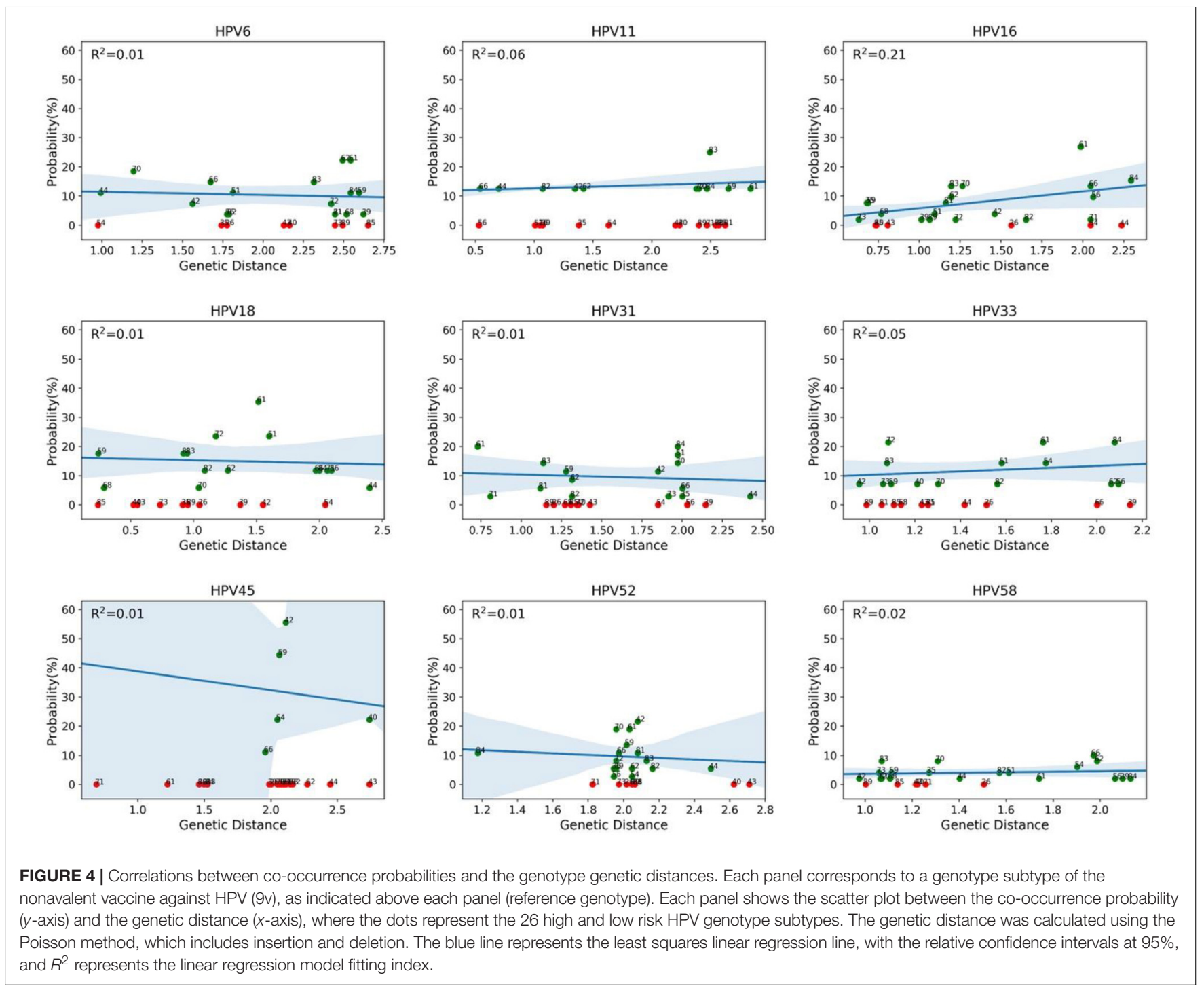

The incidence of intraepithelial lesions is 8.3 per $100 \mathrm{HIV}$-positive women per year, compared to 1.8 for HIV-negative women $(p<0.001)$ (Ellerbrock et al., 2000). Increased rates of perianal, vaginal, and vulvar squamous dysplasia are also observed for HIV-positive women (Jamieson et al., 2006). The persistence of HPV infections that are linked to impairment of the immune system represents the main risk for ICC development. In HIVpositive women, ICC arises 10-15 years sooner in comparison to HIV-negative women, and it has a worse prognosis. HAART therapy and the increase in CD4 T cell counts do not appear to influence the course of the malignancy (Joseph and Chiramana, 2004; Pantanowitz et al., 2010). The 9v has been shown to be effective to prevent infections of the genotypes included in the vaccine, but it is still debated whether it provides protection against other genotypes. This is a remarkable limitation, as the persistent HPV infections observed in HIV-positive women are caused by a broad diversity of both HR and LR types (Joseph and Chiramana, 2004; De Vuyst et al., 2008; McClymont et al., 2020). These data are confirmed also by the present study: $49 \%$ of infections were represented by non9v HPV types (Figure 1). Among these, 15 HSIL were observed, where nine were SI and six were MI. Interestingly, four SI harbored HPV61, 62, and 83, which are considered to be LR types. Twenty-one LSIL specimens were SI positive for LR risk HPV42, 54, 55, 61, 62, 72, 81, 84, and 5 MI exhibited LR HPV42, 54, 61, 62, 71, and 81 (Figure 2).

Recently, Bogaards et al. re-evaluated the effectiveness of the adjuvanted bivalent HPV vaccine (2vHPV) against type-specific HPV positivity. Here, they demonstrated that cross-protection is maintained up to 8 years post-vaccination, and that the level of protection correlates with genomic distance to HPV16 or HPV 18. Indeed they showed that $2 \mathrm{vHPV}$ can partially crossprotect against HPV types belonging to HPV $\alpha-7$ (including HPV18) and $\alpha-9$ (including HPV16), but cross-protection was unlikely for HPV51, 53, and 56, and for HR types belonging to the HPV $\alpha-5$ or $\alpha-10$ species; Bogaards et al., 2019). At variance with this, our data obtained from a real-life setting of HPV MI demonstrated that this correlation does not exist. 


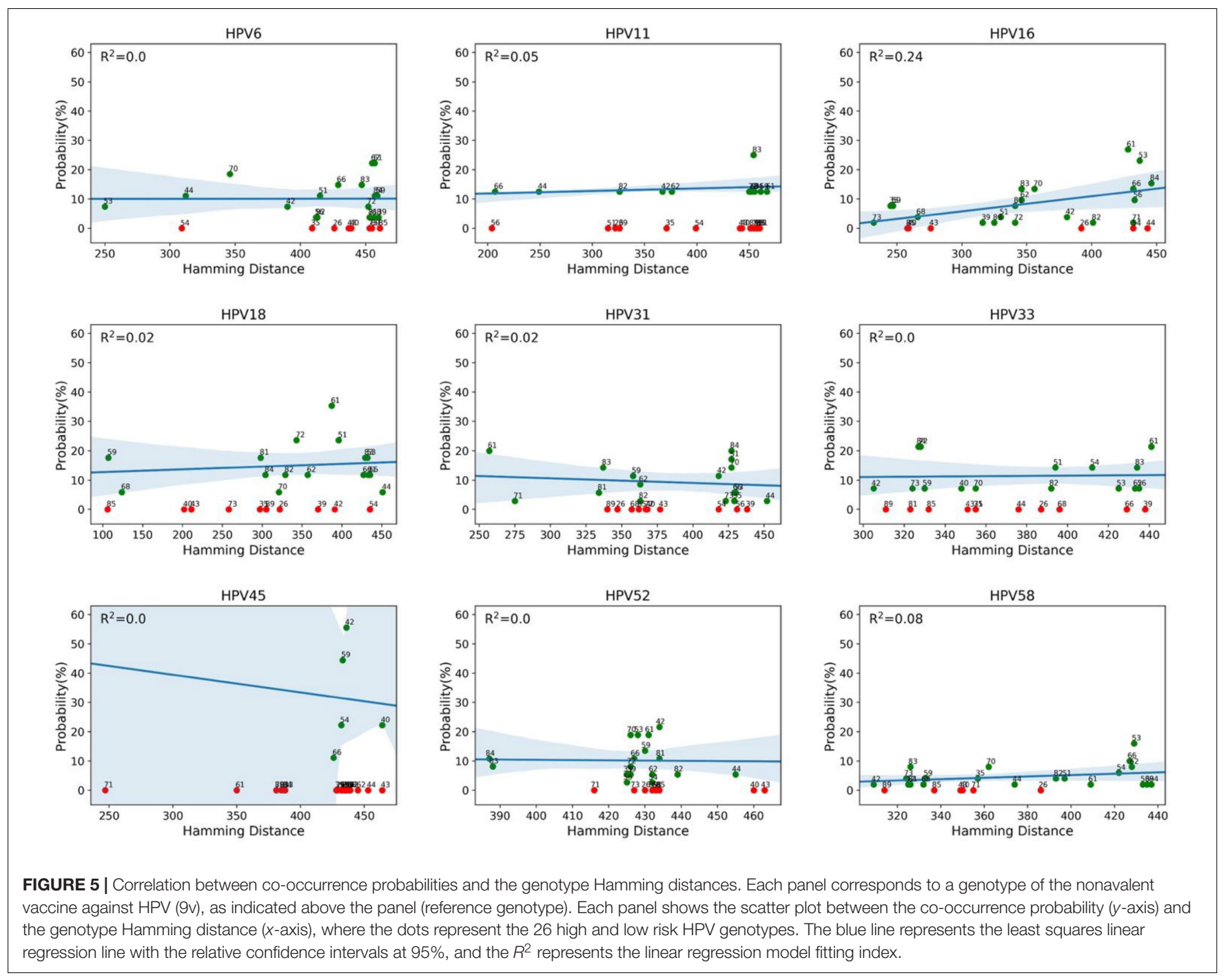

For instance, HPV16 had a similar probability to co-exist with HPV53 $(\alpha-6)$ and with HPV61 $(\alpha-3)$. A similar probability of co-infection was observed for HPV35 $(\alpha-9)$ and HPV81 $(\alpha-$ $3)$. HPV33 showed a comparable probability to co-exist with HPV56 $(\alpha-6)$ and HPV59 $(\alpha-7)$, irrespective of the genetic distance (Figures 3-6). HPV26, 35, 40, 43, 54, 73, and 85 are the genotypes that are completely protected by at least one of the $9 \mathrm{v}$ types.

A bias of this analysis might relate to the different prevalence of HPV types in different geographical areas, and thus we might have included fewer cases of an HPV type because it is not very common in Europe (e.g., HPV26, 40, 43, and 89). However, we observed that HPV types that belong to different $\alpha$-species and that have similar prevalence in Italy co-existed with 9v HPV types with similar frequencies.HPV52 $(\alpha-9)$ infection occurred with HPV59 $(\alpha-7)$ and HPV81 $(\alpha-$ 3 ), which had the same prevalence in a population of women (Annunziata et al., 2018). These findings suggest that the cross-protection between $9 \mathrm{v}$ types and other genotypes is weak or ineffective.
The absence of cross-protection against HPV types that belong to both the same and different $\alpha$-species was also seen for HPV16 and HPV18 in vaccinated women from Costa Rica (Skinner et al., 2016), where HPV31 ( $\alpha-9)$ and HPV51 $(\alpha-6)$ infections appeared post-vaccination. Moreover, considering that sublineage variants show significant differences in neutralization sensitivity (Godi et al., 2015; Godi et al., 2019), we examined HPV81 strains, to investigate whether different $L 1$ antigenic variants were present in SI and MI, and whether they might influence their cross-protection properties. Overall, the L1 protein harbored a greater number of variable amino acids than the L2 protein ( 35 amino-acid vs 9 aminoacid changes, respectively). For L1, six amino-acid mutations affected two antigenic sites: the BC loop (T84N, Pt422) and the FGb loop (T315N, Pt363, Pt422, Pt574, Pt644, and Pt798; Figure 7, Supplementary Table 1). In contrast, no amino-acid mutations occurred for L2 antigenic sites. In our analysis of the HPV81 L1 and L2 $\beta$-turns, variations were observed only in the L1 MI infection samples (Supplementary Table 1), which suggests modifications of interactions with 


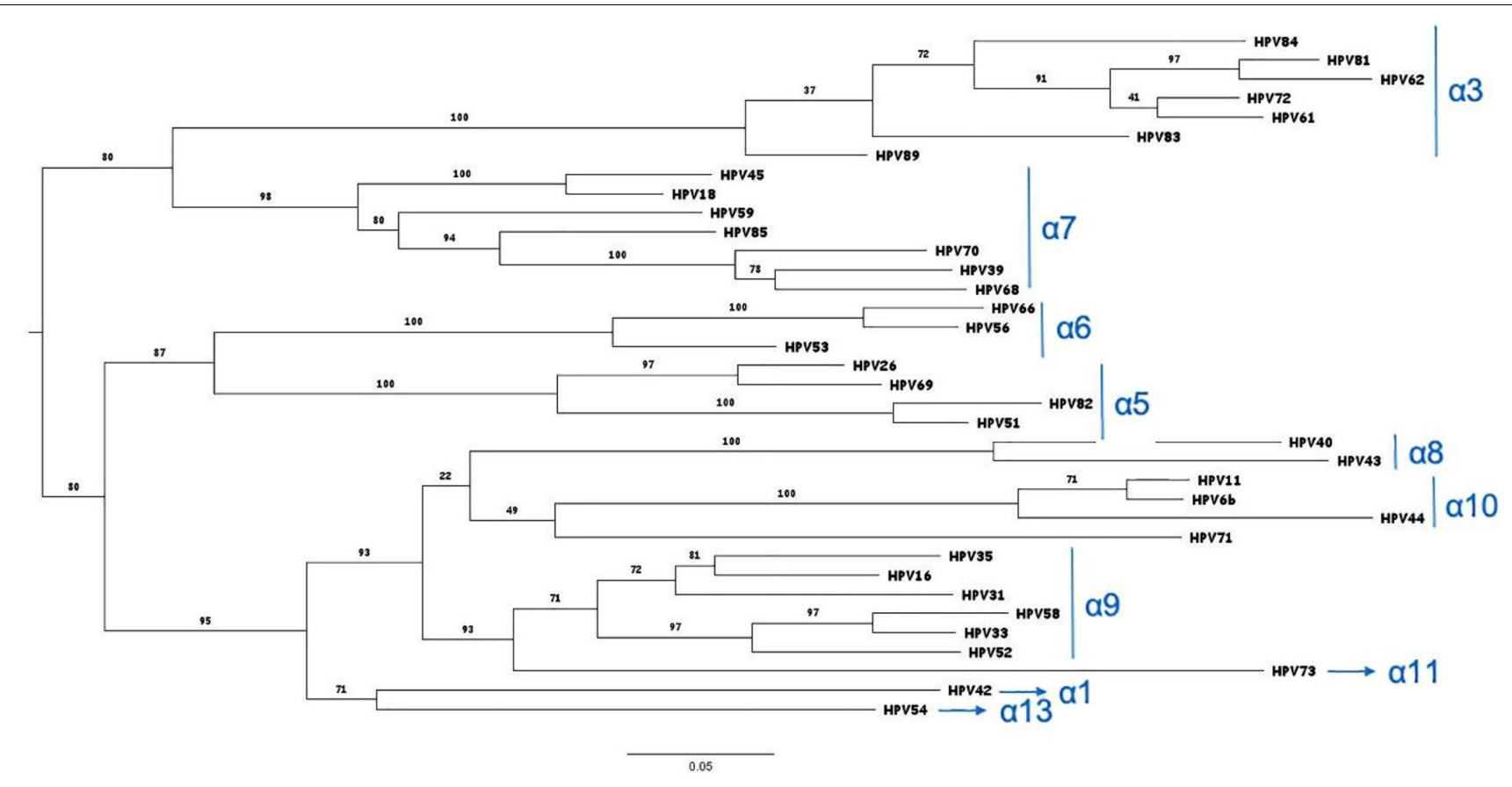

FIGURE 6 | Phylogenetic tree of the HPV genotypes detected by the HPV Genomica assay. The tree was constructed on the L1 protein sequences by the maximum likelihood method: http://www.phylogeny.fr/website (phylogeny web site). The branch support values are indicated (Anisimova and Gascuel, 2006).

antibodies and cross-reactivity between different HPV types. Indeed, several studies have demonstrated the involvement of $\beta$-turns in molecular recognition. In the immunoglobulins, the $\beta$-turns are present in the hypervariable region of CDR (Vdomain complementary determining regions), and they influence the affinity with the target antigen (Abergel et al., 1994). Single variations to $\beta$-turns impact on the conformation of proteins. Proline and glycine amino acid changes contribute to the conformational stability of $\beta$-turns (Trevino et al., 2007), and these variations were observed in L1 and MI samples. These findings suggest that the genetic differences in different settings might affect the crossneutralization sensitivity, although this assertion should be supported by further investigations extending the analysis to more variants and to other HPV low risk types, both for SI and MI. Cross-neutralization assays should demonstrate whether lower sensitivity to cross neutralizing antibodies can be related to these variants, and a follow-up study should be conducted to determine whether lower neutralization activity has clinical relevance.

As previously indicated, the data in the literature have shown limited efficacies of the HPV vaccines against non-vaccine HPV types (Innes et al., 2018; McClymont et al., 2020). This leads to consideration of the need for anti-HPV vaccine implementation. The RG-1-L2 epitope might be a candidate to reach this goal. To date, an L2-based vaccine has never been introduced into clinical practice because although it induces neutralizing antibodies against a broad spectrum of genotypes, the antibody titer is lower than that induced by the L1 protein (Chandrachud et al., 1995; Gambhira et al., 2007). RG1-L2 (amino acids, 17-36) of HPV16 was inserted into the L1 DE loop. The chimeric VLPs induced cross neutralizing antibodies that were effective against most of the HR genotypes, or potentially carcinogenic types (HPV16, 18, 45, 31, 33, 35, 52, 58, 39, 51, 59, 68, 73, 26, 53, and 66), and LR HPV6, 43, 44 (Schellenbacher et al., 2009, 2013). In another study, amino acids 17-36 of HPV33 L2 were positioned into the HPV18 L1 DE loop, and amino acids 56-75 of the HPV58 L2 protein were inserted into the L1-COOH terminus. These VLPs induced persistent immune responses against the HPV6, 11, 16, 31, 35, 39, 45, 58, and 59 genotypes in both mice and rabbits (Boxus et al., 2016). Sequence conservation of L2 proteins allows a broadly protective response to be elicited using a few epitopes (Roden et al., 2000). The HPV81 analysis in SI and MI settings confirmed the high conservation of the L2 protein, showing no variability in antigenic sites, strengthening the usefulness of the L2 protein in HPV vaccine implementation.

\section{CONCLUSION}

In summary, this study on cervical specimens from HIVpositive women showed a high prevalence of LSIL and HSIL that are related to non9v HPV types, and several of these are considered LR types. The analysis of co-infectivity starting from the real-life setting suggests that $9 \mathrm{v}$ types do not protect against other genotypes, and thus a vaccination campaign cannot fully prevent HPV-related intra-epithelial lesions. Therefore, these data confirm the importance of a sustainable and effective screening program, which should 
A

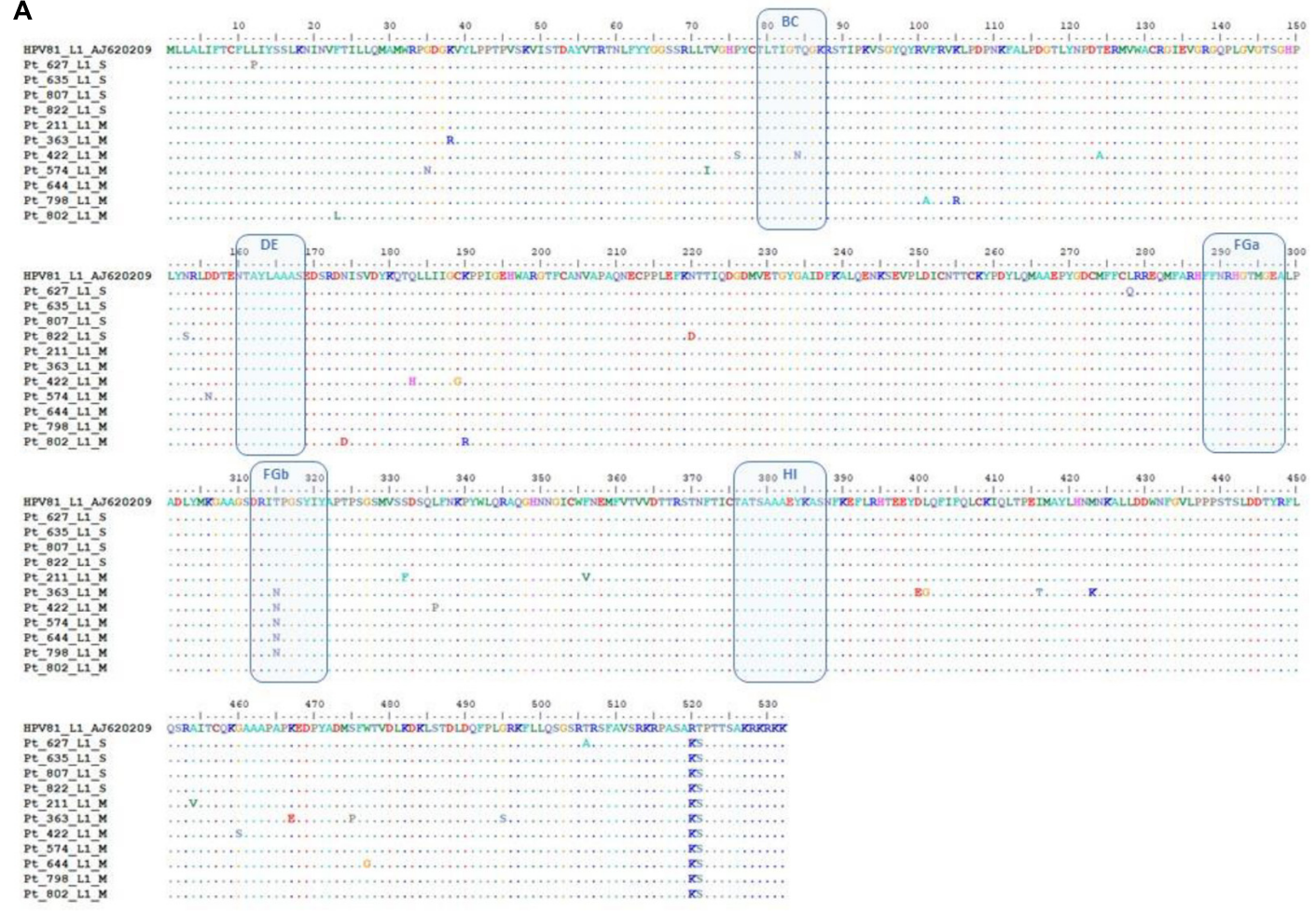

B

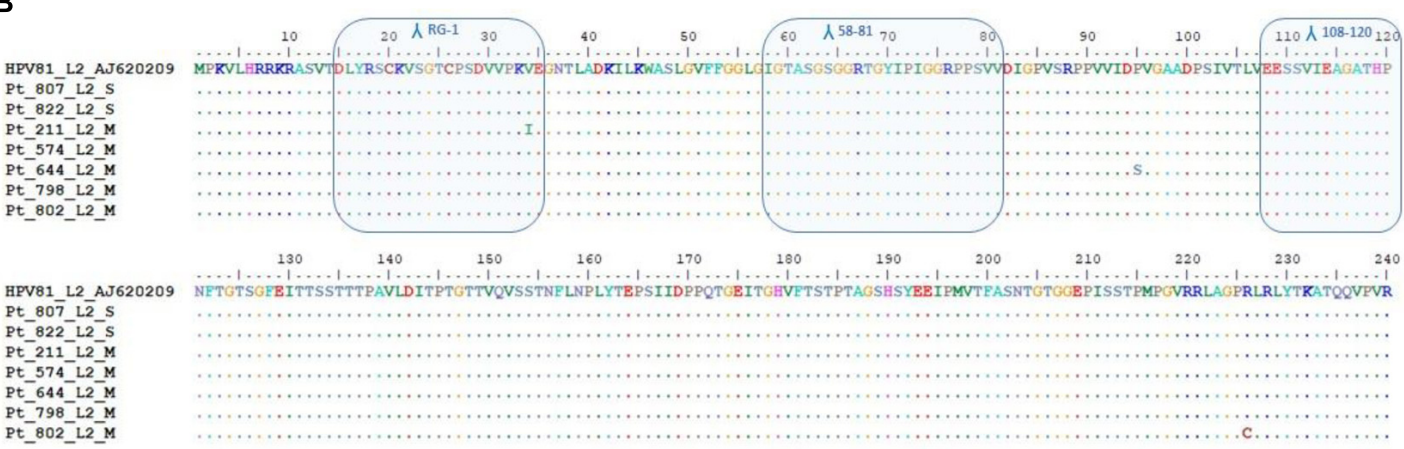

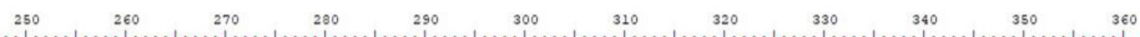

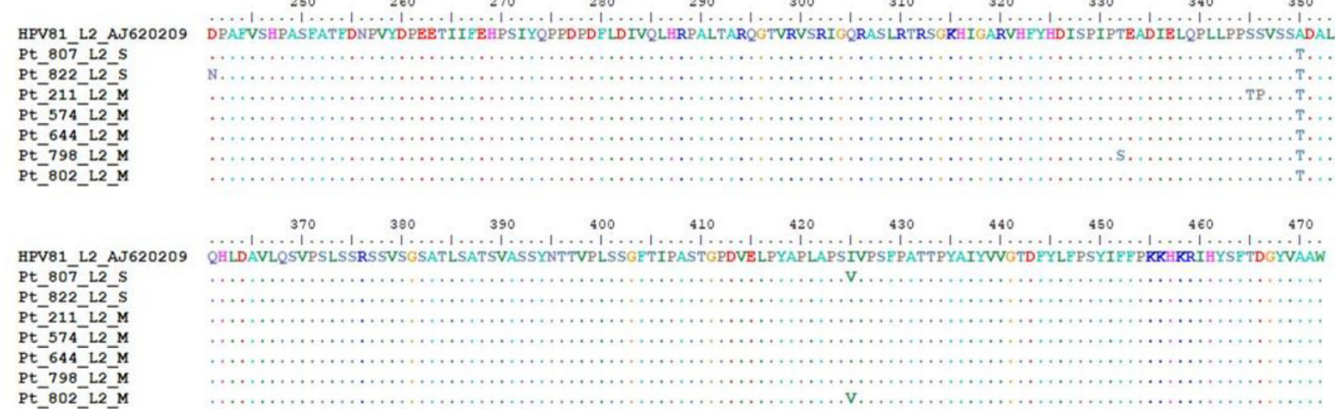

FIGURE 7 | HPV81 L1 and L2 sequence analysis. (A) Alignment of the deduced amino-acid sequences of HPV81 L1 from four patients with HPV single infection (SI) and seven patients with HPV multiple infection (MI). The BC, DE, FGa FGb, and HI immunogenic regions that are shown on the surface of the protein are indicated in boxes. (B) Alignment of the deduced amino-acid sequences of HPV81 L2 from five patients with HPV Ml and two patients with HPV SI. The patients from whom the sequences originated are indicated by their identification codes. The sequences were aligned with HPV81 prototype AJ620209. Dashes indicate amino-acid sequence identity with respect to the reference sequence. $\Lambda$, antigenic site. 
be implemented through HPV DNA testing that does not include only HR types.

\section{DATA AVAILABILITY STATEMENT}

The sequences of the L1 and L2 genes were submitted to the NCBI GenBank database, and were assigned accession numbers (MT547553-MT547561).

\section{ETHICS STATEMENT}

The studies involving human participants were reviewed and approved by Institutional Ethical Committee L Spallanzani code42/13, 2013. The patients/participants provided their written informed consent to participate in this study.

\section{AUTHOR CONTRIBUTIONS}

ARG, PDP, and PP conceived and designed the study. CS and DL collected the samples and carried out sample HPV typing. FDN processed the cythological samples. CS, VG, CM, DL, and FDN analyzed the data. ARG and VG

\section{REFERENCES}

Abergel, C., Tipper, J. P., and Padlan, E. A. (1994). Structural significance of sequence variability in antibody complementarity-determining regions. Res. Immunol. 145, 49-53. doi: 10.1016/s0923-2494(94)80043-X

Al-Lawati, Z., Khamis, F. A., Al-Hamdani, A., Al-Kalbani, M., Ramadhan, F. A., Al-Rawahi, T. R., et al. (2020). Prevalence of human papilloma virus in Oman: genotypes 82 and 68 are dominating. Int. J. Infect. Dis. 93, 22-27. doi: 10.1016/ j.ijid.2019.12.038

Amaro-Filho, S. M., Gradissimo, A., Usyk, M., Moreira, F. C. B., de Almeida, L. M., Moreira, M. A. M., et al. (2020). HPV73 a nonvaccine type causes cervical cancer. Int. J. Cancer 146, 731-738. doi: 10.1002/ijc.32315

Anisimova, M., and Gascuel, O. (2006). Approximate likelihood ratio test for branchs: a fast, accurate and powerful alternative. Syst. Biol. 55, 539-552.

Annunziata, C., Stellato, G., Greggi, S., Sanna, V., Curcio, M. P., Losito, S., et al. (2018). Prevalence of "unclassified" HPV genotypes among women with abnormal cytology. Infect. Agent. Cancer 24:26.

Bickel, P., and Doksum, K. (1977). Mathematical Statistics: basic Ideas and Selected Topics. San Francisco, CA: Holden-Day.

Bishop, B., Dasgupta, J., Klein, M., Garcea, R. L., Christensen, N. D., Zhao, R., et al. (2007). Crystal structures of four types of human papillomavirus L1 capsid proteins: understanding the specificity of neutralizing monoclonal antibodies. J. Biol. Chem. 282, 31803-31811. doi: 10.1074/jbc.m706380200

BLAST (2020). Available online at: http://blast.ncbi.nlm.nih.gov (accessed 13 Oct 2020).

Bogaards, J. A., van der Weele, P., Woestenberg, P. J., van Benthem, B. H. B., and King, A. J. (2019). Bivalent human papillomavirus (HPV) vaccine effectiveness correlates with phylogenetic distance from HPV vaccine types 16 and 19 . J. Infect. Dis. 220, 1141-1146. doi: 10.1093/infdis/jiz280

Boxus, M., Fochesato, M., Miseur, A., Mertens, E., Dendouga, N., Brendle, S., et al. (2016). Broad cross-protection is induced in preclinical models by a human papillomavirus vaccine composed of L1/L2 chimeric virus-like particles. J. Virol. 90, 6314-6325. doi: 10.1128/jvi.00449-16

Bretscher, O. (1995). Linear Algebra with Applications, 3rd Edn. Upper Saddle River, NJ: Prentice Hall. writing original draft. ARG, PDP, PP, VG, and MC edited the manuscript. All authors read and agreed to the final version of manuscript.

\section{FUNDING}

This work was supported by Ricerca Corrente Funding from the Italian Ministry of Health.

\section{ACKNOWLEDGMENTS}

This study is part of a sexually transmitted diseases prevention activity and therefore we recognize all physicians and nurses of the Clinical Department as well as the staff of the Virology laboratory for their generous contribution to patient invoice, clinical examinations, data registration, and laboratory analysis.

\section{SUPPLEMENTARY MATERIAL}

The Supplementary Material for this article can be found online at: https://www.frontiersin.org/articles/10.3389/fmicb. 2020.603657/full\#supplementary-material

Brotherton, J. M. L., Tabrizi, S. N., Phillips, S., Pyman, J., Cornall, A. M., Lambie, N., et al. (2017). Looking beyond human papillomavirus (HPV) genotype 16 and 18: defining HPV genotype distribution in cervical cancers in Australia prior to vaccination. Int. J. Cancer 141, 1576-1584. doi: 10.1002/ijc. 30871

Casella, G., and Berger, R. (2002). Statistical Inference, 2nd Edn. Pacific Grove, CA: Duxbury Advanced Series.

Chandrachud, L. M., Grindlay, G. J., McGarvie, G. M., O’Neil, B. W., Wagner, E. R., Jarrett, W. F., et al. (1995). Vaccination of cattle with the N-terminus of L2 is necessary and sufficient for preventing infection by bovine papillomavirus. Virology 211, 204-208. doi: 10.1006/viro.1995.1392

Christensen, N. D., Cladel, N. M., Reed, C. A., Budgeon, L. R., Embers, M. E., Skulsky, D. M., et al. (2001). Hybrid papillomavirus L1 molecules assemble into virus-like particles that reconstitute conformational epitopes and induce neutralizing antibodies to distinct HPV types. Virology 291, 324-334. doi: 10.1006/viro.2001.1220

Combet, C., Blanchet, C., Geourjon, C., and Deléage, G. (2000). NPS@: network protein sequence analysis. Trends Biochem. Sci. 25, 147-150. doi: 10.1016/ s0968-0004(99)01540-6

De Martel, C., Ferlay, J., Franceschi, S., Vignat, J., Bray, F., Forman, D., et al. (2012). Global burden of cancers attributable to infections in 2008: a review and synthetic analysis. Lancet Oncol. 13, 607-615. doi: 10.1016/s1470-2045(12) 70137-7

De Villiers, E. M. (2013). Cross-roads in the classification of papillomaviruses. Virology 445, 2-10. doi: 10.1016/j.virol.2013.04.023

De Vuyst, H., Gichangi, P., Estambale, B., Njuguna, E., Franceschi, S., and Temmerman, M. (2008). Human papillomavirus types in women with invasive cervical carcinoma by HIV status in Kenya. Int. J. Cancer 122, 244-246. doi: $10.1002 /$ ijc. 23045

Drolet, M., Bénard, É, Pérez, N., and Brisson, M. (2019). HPV vaccination impact study group. Population-level impact and herd effects following the introduction of human papillomavirus vaccination programmes: updated systematic review and meta-analysis. Lancet 394, 497-509.

Durrett, R. (1996). Probability: Theory and Examples, 2nd Edn. California: Duxbury Press. 
Ellerbrock, T. V., Chiasson, M. A., Bush, T. J., Sun, X. W., Sawo, D., Brudney, K., et al. (2000). Incidence of cervical squamous intraepithelial lesions in HIV-infected women. JAMA 283, 1031-1037. doi: 10.1001/jama.283.8.1031

Farci, P., Shimoda, A., Coiana, A., Diaz, G., Peddis, G., Melpolder, J. C., et al. (2000). The outcome of acute hepatitis C predicted by the evolution of the viral quasispecies. Science 288, 339-344. doi: 10.1126/science.288.5464.339

Feller, W. (1971). An Introduction to Probability Theory and its Applications, 2nd Edn, Vol. II. New York, NY: John Wiley \& Sons.

Ferlay, J., Colombet, M., Soerjomataram, I., Mathers, C., Parkin, D. M., Piñeros, M., et al. (2019). Estimating the global cancer incidence and mortality in 2018: GLOBOCAN sources and methods. Int. J. Cancer 144, 1941-1953. doi: 10.1002/ ijc. 31937

Gambhira, R., Karanam, B., Jagu, S., Roberts, J. N., Buck, C. B., Bossis, I., et al. (2007). A protective and broadly cross-neutralizing epitope of human papillomavirus L2. J. Virol. 81, 13927-13931. doi: 10.1128/jvi.00936-07

Garbuglia, A. R., Lapa, D., Sias, C., Capobianchi, M. R., and Del Porto, P. (2020). The use of both therapeutic and prophylactic vaccines in the therapy of papillomavirus disease. Front. Immunol. 18:188.

Garbuglia, A. R., Piselli, P., Lapa, D., Sias, C., Del Nonno, F., Baiocchini, A., et al. (2012). Frequency and multiplicity of human papillomavirus infection in HIV1 positive women in Italy. J. Clin. Virol. 54, 141-146. doi: 10.1016/j.jcv.2012.02. 013

Garland, S. M., Hernandez-Avila, M., Wheeler, C. M., Perez, G., Harper, D. M., Leodolter, S., et al. (2007). Quadrivalent vaccine against human papillomavirus to prevent anogenital diseases. N. Engl. J. Med. 10, 1928-1943.

Ge, Y., Mody, R. R., Olsen, R. J., Zhou, H., Luna, E., Armylagos, D., et al. (2019). HPV status in women with high-grade dysplasia on cervical biopsy and preceding negative HPV tests. J. Am. Soc. Cytopathol. 8, 149-156. doi: 10.1016/j.jasc.2019.01.001

Godi, A., Facchetti, A., Bissett, S. L., Cocuzza, C., Miller, E., and Beddows, S. (2015). Naturally occurring major and minor capsid protein variants of human papillomavirus 45 (HPV45): differential recognition by cross-neutralizing antibodies generated by HPV vaccines. J. Virol. 90, 3247-3252. doi: 10.1128/ jvi.02859- 15

Godi, A., Kemp, T. J., Pinto, L. A., and Beddows, S. (2019). Sensitivity of human papillomavirus (HPV) lineage and sublineage variant pseudoviruses to neutralization by nonavalent vaccine antibodies. J. Infect. Dis. 220, 1940-1945. doi: 10.1093/infdis/jiz401

Hacksparrow (2019). Available online at: https://www.hacksparrow.com/tools/ calculators/hamming-distance.html (accessed July 21, 2019). doi: 10.1093/ infdis/jiz401

Hamming, R. W. (1986). Coding and Information Theory, 2nd Edn. Englewood Cliffs, NJ: Prentice-Hall.

Innes, C. R., Sykes, P. H., Harker, D., Williman, J. A., Van der Griend, R. A., Whitehead, M., et al. (2018). Changes in human papillomavirus genotypes associated with cervical intraepithelial neoplasia grade 2 lesions in a cohort of young women (2013-2016). Papillomavirus Res. 6, 77-82. doi: 10.1016/j.pvr. 2018.10.010

Jamieson, D. J., Paramsothy, P., Cu-Uvin, S., Duerr, A., and HIV Epidemiology Research Study Group (2006). Vulvar, vaginal, and perianal intraepithelial neoplasia in women with or at risk for human immunodeficiency virus. Obstet. Gynecol. 107, 1023-1028. doi: 10.1097/01.aog.0000210237. 80211.ff

Joseph, B., and Chiramana, H. (2004). Extensive subcutaneous metastasis from squamous cell carcinoma of the cervix in patient with HIV. Int. J. Gynecol. Cancer 14, 176-177. doi: 10.1136/ijgc-00009577-200401000-00027

Kelley, D. (1994). Introduction to Probability. London: Macmillan Publishing Company.

Kumar, S., Stecher, G., Li, M., Knyaz, C., and Tamura, K. (2018). MEGA X: molecular evolutionary genetics analysis across computing platforms. Mol. Biol. Evol. 35, 1547-1549. doi: 10.1093/molbev/msy096

Larson, R., and Farber, B. (2003). Elementary Statistics: Picturing the World. Englewood Cliffs, NJ: Prentice-Hall.

Lopalco, P. L. (2016). Spotlight on the 9-valent HPV vaccine. Drug Des. Devel. Ther. 11, 35-44. doi: 10.2147/dddt.s91018

Luostarinen, T., Apter, D., Dillner, J., Eriksson, T., Harjula, K., Natunen, K., et al. (2018). Vaccination protects against invasive HPV-associated cancers. Int. J. Cancer 142, 2186-2187. doi: 10.1002/ijc.31231
Maiman, M. (1994). Cervical neoplasia in women with HIV infection. Oncology 8, 83-89.

matplotlib (2020). Available online at: www.matplotlib.org (accessed September 15, 2020).

McClymont, E., Coutlée, F., Lee, M., Albert, A., Raboud, J., Walmsley, S., et al. (2020). Brief report: persistence of non-vaccine oncogenic HPV genotypes in quadrivalent HPV-vaccinated women living with HIV. J. Acquir. Immune. Defic. Syndr. 83, 230-234. doi: 10.1097/qai.0000000000002258

Minosse, C., Garbuglia, A. R., Lapa, D., Sias, C., Zaniratti, M. S., and Capobianchi, M. R. (2010). Genetic variability in E6, E7 and L1 protein of HPV81 from HIV-1 positive women in Italy. New Microbiol. 33, 25-35.

Moore, D., and McCabe, G. (2003). Introduction to the Practice of Statistics. London: W. H. Freeman and Co.

Muñoz, N., Bosch, F. X., de Sanjosé, S., Herrero, R., Castellsagué, X., Shah, K. S., et al. (2003). Epidemiologic classification of human papillomavirus types associated with cervical cancer. N. Engl. J. Med. 348, 518-527. doi: 10.1056/ nejmoa021641

NumPy (2020). Available online at: www.numpy.org (accesed May 15, 2020).

Nygård, M., Saah, A., Munk, C., Tryggvadottir, L., Enerly, E., Hortlund, M., et al. (2015). Evaluation of the long-term anti-human papillomavirus 6 (HPV6), 11, 16 , and 18 immune responses generated by the quadrivalent HPV vaccine. Clin. Vaccine Immunol. 22, 943-948. doi: 10.1128/cvi.00133-15

Palefsky, J. M., Minkoff, H., Kalish, L. A., Levine, A., Sacks, H. S., Garcia, P., et al. (1999). Cervicovaginal human papillomavirus infection in human immunodeficiency virus-1 (HIV)-positive and high-risk HIV-negative women. J. Natl. Cancer Inst. 91, 226-236. doi: 10.1093/jnci/91.3.226

Pandas (2020). Available online at: https://pandas.pydata.org/ (accessed October 30, 2020)

Pantanowitz, L., Hornish, M., Cao, Q. J., and Goulart, R. A. (2010). HPV data can be used as a cytopathology laboratory quality indicator. Diagn. Cytopathol. 38, 159-160.

Park, E., Kim, J. Y., Choi, S., Kim, D. S., and Oh, Y. L. (2019). Carcinogenic risk of human papillomavirus (HPV) genotypes and potential effects of HPV vaccines in Korea. Sci. Rep. 9:12556.

Peirson, L., Fitzpatrick-Lewis, D., Ciliska, D., and Warren, R. (2013). Screening for cervical cancer: a systematic review and meta-analysis. Syst. Rev. 24:35.

Phylogeny (2020). Available online at: http://www.phylogeny.fr/ (accessed May 3, 2020).

Prabi (2020). Available online at: https://npsa-prabi.ibcp.fr/cgi-bin/npsa_automat. pl?page=/NPSA/npsa_sopm.html (accessed on April 27, 2020).

Python (2020). Available online at: www.python.org (accesed May 15, 2020).

Roden, R. B., Yutzy, W. H., Fallon, R., Inglis, S., Lowy, D. R., and Schiller, J. T. (2000). Minor capsid protein of human genital papillomaviruses contains subdominant, cross-neutralizing epitopes. Virology 270, 254-257. doi: 10.1006/ viro.2000.0272

Saiki, R. K., Scharf, S., Faloona, F., Mullis, K. B., Horn, G. T., Erlich, H. A. et al. (1985). Enzymatic amplification of beta-globin genomic sequences and restriction site analysis for diagnosis of sickle cell anemia. Science 230, 1350 1354. doi: 10.1126/science. 2999980

Schellenbacher, C., Kwak, K., Fink, D., Shafti-Keramat, S., Huber, B., Jindra, C., et al. (2013). Efficacy of RG1-VLP vaccination against infections with genital and cutaneous human papillomaviruses. Version J. Invest. Dermatol. 133, $2706-$ 2713. doi: $10.1038 /$ jid.2013.253

Schellenbacher, C., Roden, R., and Kirnbauer, R. (2009). Chimeric L1-L2 virus-like particles as potential broad-spectrum human papillomavirus vaccines. J. Virol. 83, 10085-10095. doi: 10.1128/jvi.01088-09

Scipy (2020). Available online at: www.scipy.org (accesed May 15, 2020).

Seaborn (2020). Available online at: http://seaborn.pydata.org/ (accesed May 15, 2020).

Sias, C., Garbuglia, A. R., Piselli, P., Cimaglia, C., Lapa, D., Del Nonno, F., et al. (2013). Comparison of the abbott realtime high risk HPV with genomica HPV clinical array for the detection of human papillomavirus DNA. APMIS 121, 1054-1063. doi: 10.1111/apm.12054

Sias, C., Salichos, L., Lapa, D., Del Nonno, F., Baiocchini, A., Capobianchi, M. R., et al. (2019). Alpha, Beta, gamma human PapillomaViruses (HPV) detection with a different sets of primers in oropharyngeal swabs, anal and cervical samples. Virol. J. 16:27. 
Siegler, E., Reichman, Y., Kugelman, N., Mackuli, L., Lavie, O., Ostrovsky, L., et al. (2019). Low-risk human papillomavirus types in cervical intraepithelial neoplasia 2-3 and in invasive cervical cancer patients. J. Low Genit. Tract Dis. 23, 248-252. doi: 10.1097/lgt.0000000000000486

Skinner, S. R., Apter, D., De Carvalho, N., Harper, D. M., Konno, R., Paavonen, J., et al. (2016). Human papillomavirus (HPV)-16/18 AS04-adjuvanted vaccine for the prevention of cervical cancer and HPV-related diseases. Expert Rev. Vaccines 15, 367-387. doi: 10.1586/14760584.2016.1124763

Solomon, D., Davey, D., Kurman, R., Moriarty, A., O'Connor, D., Prey, M., et al. (2002). The 2001 Bethesda system: terminology for reporting results of cervical cytology. JAMA 287, 2140-2141.

Sudenga, S. L., Rositch, A. F., Otieno, W. A., and Smith, J. S. (2013). Knowledge, attitudes, practices, and perceived risk of cervical cancer among Kenyan women: brief report. Int. J. Gynecol. Cancer 5, 895-899. doi: 10.1097/igc. $0 \mathrm{~b} 013 \mathrm{e} 31828 \mathrm{e} 425 \mathrm{c}$

Sugase, M., and Matsukura, T. (1997). Distinct manifestations of human papillomaviruses in the vagina. Int. J. Cancer 72, 412-415. doi: 10.1002/(sici) 1097-0215(19970729)72:3<412::aid-ijc7>3.0.co;2-s

Thompson, J. D., Gibson, T. J., Plewniak, F., Jeanmougin, F., and Higgins, D. G. (1997). The CLUSTAL_X windows interface: flexible strategies for multiple sequence alignment aided by quality analysis tools. Nucleic Acids Res. 25, 4876-4882. doi: 10.1093/nar/25.24.4876

Toft, L., Tolstrup, M., Müller, M., Sehr, P., Bonde, J., Storgaard, M., et al. (2014). Comparison of the immunogenicity of Cervarix ${ }^{\circledR}$ and Gardasi $l^{\circledR}$ human papillomavirus vaccines for oncogenic non-vaccine serotypes HPV-31, HPV-33, and HPV-45 in HIV-infected adults. Hum. Vaccin. Immunother. 10, 1147-1154. doi: $10.4161 /$ hv. 27925

Toh, Z. Q., Kosasih, J., Russell, F. M., Reyburn, R., Fong, J., Tuivaga, E., et al. (2019). Selective persistence of HPV cross-neutralising antibodies following reduced-dose HPV vaccine schedules. Vaccines (Basel) 7:200. doi: 10.3390/ vaccines 7040200

Trevino, S. R., Schaefer, S., Scholtz, J. M., and Pace, C. N. (2007). Increasing protein conformational stability by optimizing beta-turn sequence. J. Mol. Biol. 373, 211-218. doi: 10.1016/j.jmb.2007.07.061

Walboomers, J. M., Jacobs, M. V., Manos, M. M., Bosch, F. X., Kummer, J. A., Shah, K. V., et al. (1999). Human papillomavirus is a necessary cause of invasive cervical cancer worldwide. J. Pathol. 189, 12-19. doi: 10.1002/(sici) 1096-9896(199909)189:1<12::aid-path431>3.0.co;2-f

Weinberg, A., Song, L. Y., Saah, A., Brown, M., Moscicki, A. B., Meyer, W. A., et al. (2012). Humoral, mucosal, and cell-mediated immunity against vaccine and nonvaccine genotypes after administration of quadrivalent human papillomavirus vaccine to HIV-infected children. J. Infect. Dis. 206, 1309-1318. doi: 10.1093/infdis/jis489

Woodman, C. B., Collins, S. I., and Young, L. S. (2007). The natural history of cervical HPV infection: unresolved issues. Nat. Rev Cancer 7, 11-22. doi: $10.1038 / \mathrm{nrc} 2050$

Conflict of Interest: The authors declare that the research was conducted in the absence of any commercial or financial relationships that could be construed as a potential conflict of interest.

Copyright $\odot 2020$ Sias, Guarrasi, Minosse, Lapa, Nonno, Capobianchi, Garbuglia, Del Porto and Paci. This is an open-access article distributed under the terms of the Creative Commons Attribution License (CC BY). The use, distribution or reproduction in other forums is permitted, provided the original author $(s)$ and the copyright owner(s) are credited and that the original publication in this journal is cited, in accordance with accepted academic practice. No use, distribution or reproduction is permitted which does not comply with these terms. 